\title{
Efectos de un herbicida a base de glifosato sobre hematíes de Prochilodus lineatus (Pisces, Prochilodontidae)
}

\author{
Caramello, C.S. ${ }^{1}$; Hernandez, D.R. ${ }^{1}$; Jorge, M.J. ${ }^{2}$; Jorge, L.C. ${ }^{1}$ \\ ${ }^{1}$ Facultad Ciencias.Veterinarias, ${ }^{2}$ Fac.Cs.Exactas, Univ.Nac.del Nordeste, \\ Cabral 2139, Corrientes, Argentina. E-mail: ccaramello@vet.unne.edu.ar
}

\begin{abstract}
Resumen
Caramello, C.S.; Hernandez, D.R.; Jorge, M.J.; Jorge, L.C.: Efectos de un herbicida a base de glifosato sobre hematíes de Prochilodus lineatus (Pisces, Prochilodontidae). Rev. vet. 29: 2, 123-127, 2018. En la actualidad el sistema agrícola tiene como principal propósito el aumento de la producción con el fin de garantizar el abastecimiento y distribución de sus productos en el mercado, sea interno o externo, generando así mayor rentabilidad económica. Esto trae aparejado la implementación de diferentes agroquímicos que, vertidos al ambiente, pueden tener un impacto negativo sobre la flora, fauna, cuerpos hídricos e incluso en seres humanos. El glifosato (Roundup Full II) es uno de los herbicidas más empleados y sus beneficios están siendo objetados por las desventajas que podría provocar su utilización en forma excesiva. El objetivo de este trabajo fue evaluar la ocurrencia de alteraciones citoplasmáticas en eritrocitos de sábalo (Prochilodus lineatus) expuestos en un ensayo crónico al herbicida glifosato. Se utilizaron ocho ejemplares juveniles de sábalo, los que fueron distribuidos aleatoriamente en dos tratamientos según la adición del herbicida: control (C) sin adición y tratado (T) con adición de $0,1 \mu \mathrm{g} / 1$ de glifosato. El ensayo tuvo una duración de 70 días. Para la preparación de los frotis sanguíneos se procedió a la extracción de sangre periférica por punción de la vena caudal. Las alteraciones observadas fueron presencia de vacuolas citoplasmáticas, dacriocitosis y microcitosis. Estas anormalidades se presentaron con mayor frecuencia en el grupo $\mathrm{T}$ con respecto al C. Los resultados obtenidos sugieren la existencia de una respuesta patológica a nivel hemático ante una exposición crónica a la dosis de $0,1 \mu \mathrm{g} / \mathrm{l}$ del herbicida.
\end{abstract}

Palabras clave: peces, plaguicida glifosato, citotoxicidad, alteraciones eritrocitarias.

\begin{abstract}
Caramello, C.S.; Hernandez, D.R.; Jorge, M.J.; Jorge, L.C.: Effects of a glyphosatecontaining formulation on erythrocytes of Prochilodus lineatus (Pisces, Prochilodontidae). Rev. vet. 29: 2, 123-127, 2018. Nowadays, agriculture main purpose is to increase production in order to guarantee the supply and distribution of products to both national and international markets, thus improving economic profitability. The latter is accompanied by the use of different agrochemicals that, discharged to the environment, can have a negative impact on flora, fauna, water bodies and even on humans. Glyphosate is a widely used herbicide which benefits are being questioned due to the disadvantages related to its excessive use. The objective of this work was to evaluate the occurrence of cytoplasmic alterations in "sábalo" erythrocytes (Prochilodus lineatus) exposed to a chronic test with glyphosate (Roundup Full II). Eight "sábalo" juvenils were used which were randomly distributed into two treatments: control (C), without glyphosate, and treated (T), with the addition of $0.1 \mu \mathrm{g} / \mathrm{l}$ of the herbicide. The trial lasted 70 days. For the preparation of blood smears, peripheral blood was collected by puncture of the caudal vein. The alterations observed were cytoplasmic vacuoles, dacryocytosis and microcytosis. These abnormalities occurred more frequently in the $\mathrm{T}$ group compared to C group. Results suggest the existence of an hematic pathological response when "sábalos" were submitted to a chronic exposure of $0.1 \mu \mathrm{g} / \mathrm{l}$ of the herbicide.
\end{abstract}

Key words: fishes, herbicide glyphosate, cytotoxicity, erythrocytic alterations.

\section{INTRODUCCIÓN}

El uso creciente e inapropiado de agroquímicos para proteger las cosechas genera desechos que po-

Recibido: 16 marzo 2018 / Aceptado: 23 mayo 2018 drían contaminar a los trabajadores involucrados en la manipulación del pesticida y además acumularse en el ambiente afectando directa e indirectamente a todos los seres vivos, suelos y cuerpos de agua ${ }^{12,17}$. En la actividad agrícola de Argentina, uno de los plaguicidas ampliamente difundido es el Roundup Full II, cuyo 
compuesto activo es el glifosato ( $\mathrm{N}$-fosfonometil glicina).

El glifosato es un herbicida no selectivo de amplio espectro ${ }^{1}$, de carácter ácido, con toxicidad clase II (moderadamente tóxico) según la categorización de la Agencia de Protección Ambiental de los Estados Unidos ${ }^{28}$. Este herbicida afecta todos los órganos de la planta y se aplica sobre el follaje en la etapa de postemergencia ${ }^{25}$.

Los cuerpos de agua son los principales receptáculos de los efluentes provenientes de diversas actividades ${ }^{18}$. Por ello, es lógico pensar que estas sustancias podrían acumularse en la fauna acuática y en los sedimentos.

Los peces son utilizados como organismos bioindicadores de toxicidad ambiental, por ser muy sensibles y útiles en la evaluación de riesgos ecológicos por contaminantes químicos, ya que interactúan en varios niveles de la cadena trófica ${ }^{13,23}$, por lo cual pueden sufrir bioacumulación de contaminantes en sus tejidos y las posibles lesiones podrían ser detectadas mediante la implementación de biomarcadores ${ }^{14}$.

Los biomarcadores son definidos como respuestas biológicas provocadas por los contaminantes, dando lugar a la identificación y categorización de los daños producidos en los tejidos ${ }^{15}$. Las alteraciones citoplasmáticas en los hematíes de los teleósteos son biomarcadores de exposición a agentes tóxicos y exhiben los efectos de los mismos como respuestas a dicha exposición.

Este tipo de biomarcador tiene la ventaja de permitir el examen sobre las células sanguíneas, tanto en condiciones de laboratorio como a campo y representa un elemento eficaz para la detección de los efectos causados por agroquímicos. Diversas investigaciones con pesticidas han evaluado el daño a nivel celular y detectaron alteraciones citoplasmáticas de eritrocitos de teleósteos como respuesta biológica provocada por la exposición a los contaminantes ${ }^{2-4,16,20,22,26}$.

Por lo expuesto, el objetivo de este trabajo fue evaluar la ocurrencia de alteraciones citoplasmáticas en eritrocitos de sábalos (Prochilodus lineatus) expuestos en un ensayo crónico al herbicida glifosato.

\section{MATERIAL Y MÉTODOS}

Los procedimientos metodológicos empleados fueron avalados por el Comité de Ética y Bioseguridad de la Facultad de Ciencias Veterinarias, Universidad Nacional del Nordeste (UNNE), según Protocolo 0033.

En el ensayo se utilizaron ejemplares juveniles de Prochilodus lineatus (Characiformes, Prochilodontidae, Valenciennes 1847), provenientes del Centro de Piscicultura del Instituto de Ictiología del Nordeste (INICNE), Facultad de Ciencias Veterinarias, UNNE. Los peces fueron sometidos a un período de aclimatación durante una semana antes del ensayo, siendo alimentados con ración apropiada cada $48 \mathrm{~h}$. Durante la aclimatación los especimenes se mantuvieron en tan- ques de 3001 de agua a $20^{\circ} \mathrm{C}, \mathrm{pH} 7,0$ y aireación constante. El fotoperíodo fue ajustado a $12 \mathrm{~h}$ de oscuridad y $12 \mathrm{~h}$ de luz.

Posteriormente, ocho especimenes de $P$. lineatus fueron colocados individualmente en acuarios de 251 de capacidad cargados con 201 de agua; cuatro de ellos fueron asignados al grupo control (C) y el resto al grupo tratado (T). Los acuarios del grupo C recibieron solo agua de pozo artesiano, el grupo $\mathrm{T}$ además del agua recibió $0,1 \mathrm{ug} / 1$ de glifosato.

El ensayo tuvo una duración de 70 días. Transcurrido este período de experimentación los peces fueron anestesiados con metasulfonato de tricaína MS-222 (Finquel $\left.{ }^{\circledR}\right)$ disuelto en agua. La extracción de sangre periférica se realizó por punción de la vena caudal utilizando jeringas heparinizadas. Para la preparación de los frotis sanguíneos se siguió la técnica convencional para ambos grupos ${ }^{10,24}$.

Se analizaron 2.000 eritrocitos en cada individuo. Solo se consideraron las células con membrana citoplasmática intacta. Finalmente se clasificaron los hematíes según su morfología. Las microfotografías fueron tomadas con cámara digital Motic 9.0 adaptada a un microscopio Olympus BX41. Las diferencias en las frecuencias de alteraciones citoplasmáticas se determinaron mediante la comparación del grupo tratado con el grupo control por medio del test no paramétrico $\mathrm{U}$ de Mann-Whitney. Para el análisis se utilizó el programa estadístico SPSS.

\section{RESULTADOS Y DISCUSIÓN}

Se analizaron por cada animal 2.000 células con membrana citoplasmática intacta, contándose un total de 8.000 tanto para el grupo control como para el tratado. En T se observaron 102 eritrocitos $(1,28 \%)$ con anomalías citoplasmáticas, mientras que en $\mathrm{C}$ se evidenció un total de $21(0,26 \%)$ (Tabla 1). Las alteraciones citoplasmáticas encontradas fueron: vacuolización (C: $10=47,63 \%$; T: $27=26,46 \%)$; microcitosis $(\mathrm{C}: 9=$ 42,85\%; T: $32=31,37 \%$ ) y dacriocitos (C: $2=9,52 \%$; T: $43=42,15 \%$ ), como se observa en Figura 1 y Tabla 2.

Estos resultados revelan que especimenes de $P$. lineatus expuestos a glifosato a la dosis de $0,1 \mu \mathrm{g} / \mathrm{l}$ muestran un aumento en la frecuencia de alteraciones citoplasmáticas contrastando con las del grupo control. El análisis estadístico de los datos evidenció una asociación entre la frecuencia de anomalías con el tipo de tratamiento $(p<0,05)$, como se detalla en la Figura 2.

Diferentes grados de lesiones hematológicas han sido descriptas en anfibios y mamíferos expuestos a glifosato ${ }^{16}$. En igual sentido, ejemplares de peces como carpas (Cyprinus carpio) sometidas a dosis subletales de glifosato, exhibieron eritrocitos con una cantidad regular de vacuolas citoplasmáticas, las cuales son consideradas alteraciones severas ya que estarían indicando un elevado grado de toxicidad hemática ${ }^{2}$.

Por otro lado, se han detectado anomalías sanguíneas en ensayos con diferentes plaguicidas utilizando 
a los peces como indicadores de contaminación ambiental. Así en C. carpio se identificaron eritrocitos crenados con evidentes alteraciones a nivel citoplasmático en los individuos tratados con concentraciones subletales de fenvalerato $(0,01 \mathrm{ppm})$ y cipermetrina $(0,02 \mathrm{ppm})$ durante un ensayo de 48 horas ${ }^{20}$.

Especímenes de Clarias batracus tratados en ensayos subagudos y a concentraciones subletales de carbaryl y carbofuran presentaron eritrocitos con alteraciones morfológicas (equinocitos) ${ }^{26}$. Ensayos con la misma especie expuestos durante $96 \mathrm{~h}$ a diferentes concentraciones de los pesticidas 2,4 D y Butaclor, provocaron distintas anomalías en el citoplasma de los hematíes, tales como equinocitos, anisocromacia, anisocitosis y vacuolas ${ }^{4}$.

Ejemplares de Chana punctatus sometidos durante 30 días a dosis de $0,05,0,10,0,15,0,20$ y $0,25 \mathrm{mg} / 1$ de malatión, exhibieron glóbulos rojos con alteraciones citoplasmáticas descriptas como acantocitos, esferocitosis, equinocitosis, vacuolización, contracciones elíp-

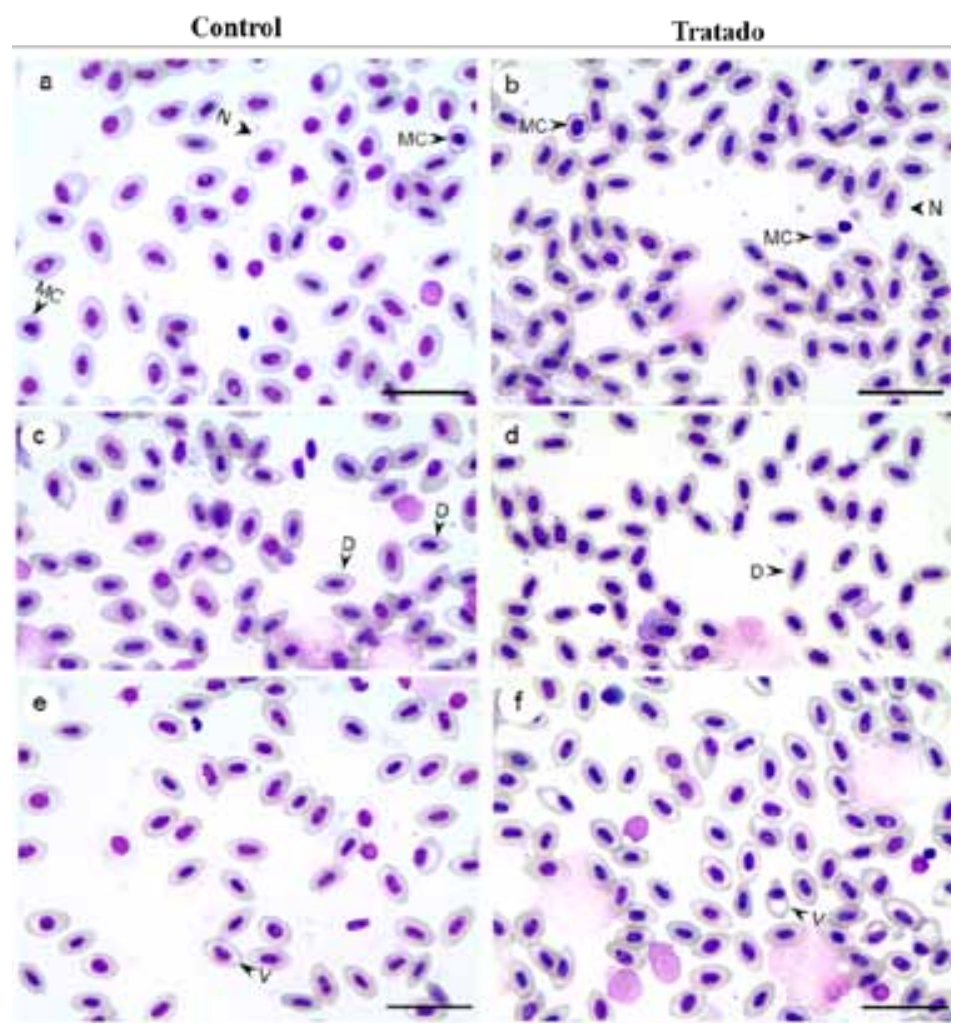

Figura 1. Microfotografía de frotis sanguíneos de Prochilodus lineatus, observándose morfología eritrocitaria de los grupos control (a, c, e) y tratado con $0,1 \mathrm{ug} / \mathrm{l}$ de glifosato (b, d, f). N: eritrocitos normales, $\mathrm{MC}$ : microcitos, $\mathrm{D}$ : dacriocitos, $\mathrm{V}$ : vacuolas citoplasmáticas. Barras $=25 \mu \mathrm{m}$. ticas de la membrana y disrupción de la misma ${ }^{22}$. Individuos de Catla catla puestos en contacto a dosis subletales de monocrotofos (insecticida), butaclor (herbicida) y radiación, revelaron diversas malformaciones como ser equinocitosis, anisocromacia, presencia de vacuolas citoplasmática y microcitosis ${ }^{3}$.

La membrana del eritrocito está constituida por un glicocálix (con abundantes carbohidratos), una bicapa lipídica (de proteínas transmembranales y lípidos) y un citoesqueleto membranal (proteínas con disposición de red, intrincada en la bicapa lipídica). El glifosato actuaría modificando la constante dieléctrica del disolvente (agua) que rodea la célula, disminuyendo las fuerzas hidrofóbicas entre los lípidos asociados 9, 19, 28 , originando un desacople en la fosforilación oxidativa $^{28}$. Tales alteraciones originan daños a nivel de la capa fosfolipídica y pérdida de la estabilidad de membrana, generándose así cambios morfológicos en los glóbulos rojos ${ }^{11}$.

Además, se demostró que la exposición a glifosato provocaría lesiones en las membranas celulares y daños en el citoesqueleto, modificaciones que estarían relacionadas con liberación citosólica de componentes, así como pérdida de la regulación iónica. En el mismo sentido, las células expuestas a glifosato evidencian cambios característicos de procesos apoptóticos ${ }^{8}$.

La presentación de dacriocitos y microcitos en sangre podría ocurrir por restricción severa de hierro, fragmentación celular y daños oxidativos a nivel de la membrana celular. En peces, dichos mecanismos serían inducidos como consecuencia de la interacción con agentes estresores 


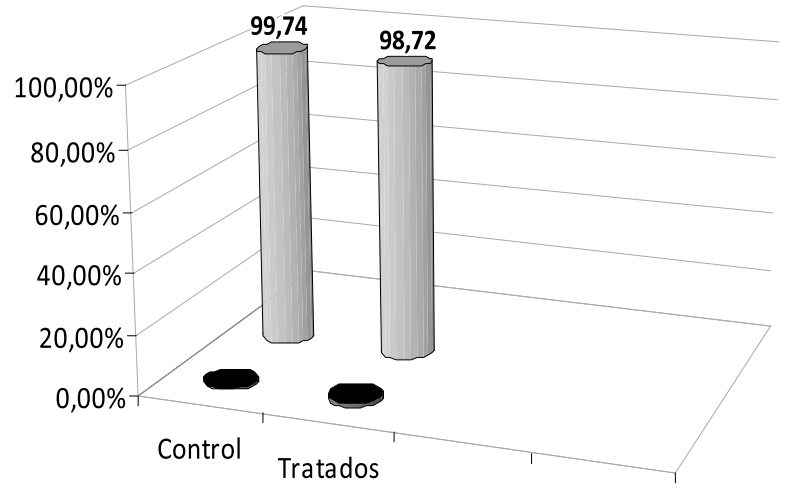

Figura 2. Incidencia de alteraciones citoplasmáticas (\%) en eritrocitos de sangre periférica de $P$. lineatus de los grupos control y tratado.

En negro: con alteraciones citoplasmáticas; en gris: sin alteraciones.

(elementos tóxicos). Diversas sustancias químicas son vertidas al agua, contaminando los recursos hídricos y afectando los organismos acuáticos. Algunas de ellas son capaces de promover alteraciones fisiológicas, incluidos los disturbios hematológicos. Tales mecanismos son desencadenados con el propósito de subsanar lesiones o adaptar el organismo a las nuevas condiciones ambientales ${ }^{6}$.

Diferentes autores sugieren que el fenómeno de vacuolización podría ocurrir como un mecanismo del organismo para disminuir la disponibilidad de xenobióti$\cos ^{7,21}$. Además, se propone que este fenómeno puede ser el resultado de daños a nivel mitocondrial ${ }^{27}$. Dichas lesiones en eritrocitos también estarían asociadas a la interacción entre el material genético y las especies de oxígeno reactivo generadas por el metabolismo del xenobiótico ${ }^{5}$.

La información obtenida en esta experiencia nos lleva a concluir que ante una exposición crónica del herbicida glifosato (70 días a la dosis de $0,1 \mu \mathrm{g} / \mathrm{l}$ ), los especimenes de $P$. lineatus exhiben una respuesta patológica a nivel eritrocitario, expresada por la formación de microcitos y dacriocitos, así como fenómenos de vacuolización. Al mismo tiempo, se destaca que los hematíes de teleósteos son excelentes indicadores de citotoxicidad, factibles de ser utilizados en el monitoreo ambiental.

\section{REFERENCIAS}

1. Alibhai MF, Stallings WC. 2001. Closing down on glyphosate inhibition with a new structure for drug discovery. Proceed Nat Acad Sci 98: 2944-2946.

2. Alvarez M, Gimenez IT, Saitua H, Enriz RD, Giannini FA. 2012. Toxicidad en peces por herbicidas formulados con glifosato. Acta Toxic Arg 20: 5-13.

3. Anbumani S, Mohankuma M. 2011. Nuclear and cytoplasmic abnormalities in the fish Catla catla (Hamilton) exposed to chemicals and ionizing radiation. Res J Envir Sci 5: 867-877.
4. Ateeq B, Farah MA, Ali MN, Ahmad W. 2002. Induction of micronuclei and erythrocyte alteration in catfish Clarias batrachus by 2,4-dichlorophenoxy acetic acid and butachlor. Mutation Res 518: 135-144.

5. Çavas T, Könen S. 2007. Detection of cytogenetic and DNA damage in peripheral erythrocytes of goldfish (Carassius auratus) exposed to a glyphosate formulation using the micronucleus test and the comet assay. Mutagenesis 22: 263-268.

6. Conroy G, Conroy DA. 2004. Patología de tilapias: uma reseña general. En: Sanidade de organismos aquáticos (Ranzani MJ, Takemoto RM, Lizama MA, Ed.), Varela Edit., São Paulo, p. 121-142.

7. Cooley HM, Evans RE, Klaverkamp JF. 2000. Toxicology of dietary uranium in lake whitefish (Coregonus clupeaformis). Aquat Toxicol 48: 495-515.

8. Eile C, Heu C, Guyon C, Nicod L. 2010. Morphological damages of glyphosate-treated human keratinocyte cell line revealed by a micro- to nanoscale microscopic investigation. Cell Biol Toxicol 26: 331-339.

9. Fonseca LC, Corrêa NC, Garrote MS, Cunha CC, Penha N. 2006. Efeito da composição do solvente sobre a estabilidade de proteínas em soluções aquosas. Quím Nova 29: 543-548.

10. Heddle JA. 1973. A rapid in vivo test for chromosomal damage. Mutation Res 18: 187-190.

11. Kalair JS, Mishra VS, Singh RK. 1993 Pesticide sumithion induced haematological changes in "mud eel" Amphipnous cuchia. Biol Membr 19: 41-48.

12. Klemz C. 2002. Uso de biomarcadores de contaminação ambiental em peixes Ancistrus sp (Cascudo). Dissertação de Mestrado, Ed. Universidade Federal do Paraná, Curitiba, Brasil, $67 \mathrm{p}$.

13. Lushchak V, Kubrak OI, Storey JM, Storey KB, Lushchak VI. 2009. Low toxic herbicide Roundup induces mild oxidative stress in goldfish tissues. Chemosphere 76 : 932-937.

14. Mana GK, Banerjee G, Gupta S. 1985. Micronucleus test in the peripheral erytrocytes of the exotic fish, Oreochromis mossambica. The nucleus 28: 176-179.

15. Martinez CB, Colus IM. 2002. Biomarcadores em peixes neotropicais para monitoramento da poluição aquática na bacia do rio Tibagi. In: A bacia do Rio Tibagi (Medri M.E. Ed.), Londrina, Paraná, Brasil, p.551-577.

16. McComb B, Curtis L, Chambers C, Newton M, Bentson K. 2008. Acute toxic hazard evaluation of glyphosate herbicide on terrestrial vertebrates of the Oregon Coast Range. Environm Sci \& Pol Res 15: 266-272.

17. Moreira JC et al. 2002. Integrated evaluation of the health impact of pesticide use in a community at Nova Friburgo, RJ. Ciência e Saúde Coletiva 7: 299-311.

18. Oliveira CA, Pelletier E, Pfeiffer WC, Rouleau C. 2000. Comparative uptake, bioaccumulation, and gill damages of inorganic mercury in tropical and nordic freshwater fish. Environm Res 83: 286-292.

19. Penha N et al. 2008. Effects of glycerol and sorbitol on the thermal dependence of the lysis of human erythrocytes by ethanol. Bioelectrochemistry 73: 23-29. 
20. Reddy PM, Bashamohideen MD. 1989. Fenvalerate and cypermethrin induced changes in the hematological parameters of Cyprinus carpio. Acta Hydrochim Hydrobiol 17: 101-107.

21. Sarkar B, Chatterjee A, Adhikari S, Ayyappan S. 2005. Carbofuran and cypermethrin induced histopathological alterations in the liver of Labeo rohita (Hamilton) and its recovery. $J$ Appl Ichthyol 21: 131-135.

22. Sawhney AK, Johal MS. 2000. Erythrocyte alterations induced by malathion in Channa punctatus (Bloch). Bull Environ Contam \& Toxicol 64: 398-405.

23. Slooff W, Dezwart D, Kerkhoff JF. 1983. Monitoring the rivers Rhine and Meuse in the Netherlands for toxicity. Aquat Toxicol 4: 189-198.

24. Schmid W. 1975. The micronucleus test. Mutat Res 31: 9-15
25. Solomon KR, Anadón KA, Cerdeira A, Marshall J, Sanín LH. 2005. Estudio de los efectos del programa de erradicación de cultivos ilícitos mediante la aspersión aérea con el herbicida glifosato. Com. Interam. Control Abuso Drogas (CICAD-OEA), Washington DC, $143 \mathrm{p}$.

26. Swarnlata S. 1995. Toxicity and fate of carbamate pesticides on blood constituents of a freshwater fish Clarias batrachus (Linn.). PhD Thesis, Avadh University, Faizabad, India.

27. Szarek J, Siwicki A, Andrzejewska A, Terech ME, Banaszkiewiez T. 2000. Effect of the herbicide Roundup on the ultrastructural pattern of hepatocytes in carp (Cyprinus carpio). Marine Environ Res 50: 263-266.

28. World Health Organization. 1994. Glyphosate, environmental health criteria $\mathrm{N}^{\circ} 159$, Geneva: WHO. On line: www.inchem.org/documents/ehc/ehclehc159.htm

\section{Asociación Cooperadora de la Facultad de Ciencias Veterinarias Universidad Nacional del Nordeste}

Personería Jurídica No 647/92 y 912/00

Sargento Cabral 2139

3400 Corrientes

Argentina

La Asociación Cooperadora de la Facultad de Ciencias Veterinarias de la UNNE fue constituida el 10 de diciembre del año 1991 como entidad de bien público, con el objeto de promover y coadyuvar las actividades científicas, educativas y culturales relacionadas con las Ciencias Veterinarias. En tal sentido, implementa acciones para colaborar con la enseñanza, extensión, actualización y difusión científica que realiza dicha casa de estudios.

\section{Beneficios que brinda a sus asociados:}

- Fotocopias con descuentos especiales en la Fotocopiadora Copias.com que funciona dentro del predio de la Facultad de Ciencias Veterinarias.

- Descuentos para la adquisición de libros de distintas editoriales.

- Descuentos especiales en otros rubros.

Tel. (0379) 4425753 interno 186•Página Web www.vet.unne.edu.ar•E-mail: cooperadora@vet.unne.edu.ar 